\title{
Psicomotricidad, corporalidad, género y filosofía positivista en Chile: análisis crítico de documentos ministeriales \\ Psychomotricity, corporeality, gender and positivist philosophy in Chile: a critical analysis of ministerial documents \\ *Loreto Chihuailaf Vera, **Felipe Nicolás Mujica Johnson, ***Rocío Fernanda Concha López \\ *Universidad de Barcelona (España), **Universidad Autónoma de Chile (Chile), ***Universidad Católica de Temuco
} (Chile)

\begin{abstract}
Resumen: La educación formal puede ser entendida como una actividad cultural que es influenciada por múltiples perspectivas epistemológicas que están presentes en la sociedad. Además, cada nación tiene sus propias particularidades en función de su propia historia y ciudadanía que son únicas. Unas de las corrientes teóricas que ha tenido un auge pedagógico en los últimos años en América Latina es la perspectiva feminista, promoviendo la igualdad de género. Considerando dicho contexto, se desarrolló el presente ensayo cuyo objetivo es analizar desde una perspectiva crítica y de género cuatro documentos ministeriales chilenos sobre psicomotricidad y corporalidad en edad infantil. Los análisis reflejaron que existe una marcada tendencia conductista, positivista, neoliberal y acrítica en torno al género, la psicomotricidad y la corporalidad en los textos ministeriales considerados. De este modo, se estaría promoviendo una educación infantil que asume una actitud pasiva ante las injusticias sociales y los estereotipos de género de corte patriarcal. En este sentido, se estaría reproduciendo, en el profesorado y en el alumnado infantil, una racionalidad curricular técnica y tradicional.
\end{abstract}

Palabras clave: igualdad de género, inclusión educativa, educación infantil, cultura patriarcal, corporalidad.

\begin{abstract}
Formal education can be understood as a cultural activity that is influenced by multiple epistemological perspectives that are present in society. In addition, each nation has its own particularities based on its own unique history and citizenship. One of the theoretical currents that has had a pedagogical boom in recent years in Latin America is the feminist perspective, promoting gender equality. Considering this context, this essay was developed with the objective of analyzing from a critical and gender perspective four Chilean ministerial documents on psychomotricity and corporeality in early childhood. The analyses showed that there is a marked behaviourist, positivist, neoliberal and uncritical tendency towards gender, psychomotor skills and corporeality in the ministerial texts considered. In this way, it would be promoting an early childhood education that assumes a passive attitude towards social injustices and patriarchal gender stereotypes. In this sense, a technical and traditional curricular rationality is being reproduced among teachers and children.
\end{abstract}

Key words: gender equality, educational inclusion, children's education, patriarchal culture, corporeality.

\section{Introducción}

Los desafíos hacia una educación emancipadora y crítica, requieren la reflexión de los mandatos culturales que subordinan las identidades en torno al orden patriarcal, neoliberal y positivista establecido (Bourdieu, 2000; Cobo, 2008; Freire, 2011; Giroux, 1990; González, 2005; Mujica, 2019; Oliva \& Gascón, 2016). Con ello, se hace referencia a que, desde el nacimiento, las personas interactúan con la sociedad e interiorizan patrones culturales de un modo acrítico (Lévi-Strauss, 1995, 2019).Y, por lo mismo, es fundamental que la educación promueva una reflexión crítica sobre aquellos significa-

Fecha recepción: 10-10-21. Fecha de aceptación: 14-12-21

Felipe Nicolás Mujica Johnson

felipe.mujica@uautonoma.cl dos interiorizados.

En nuestras sociedades la disparidad y el establecimiento de géneros binarios está bastante normalizado. Desde que una vida está en proceso de gestación su sexo biológico determinará, de algún modo, su expresión de género en la sociedad. Sus progenitores o cuidadores directos, en buena parte de los casos, generarán una especie de adhesión a un género, el cual estará determinado por colores de ropa, diseños, elección de juguetes, entre otros elementos. De tal modo, que el infante parezca en la sociedad un niño o una niña. Así, el género influirá en el desarrollo conductual, intelectual y socioafectivo de la infancia, dentro del estándar social, clasificando las conductas en masculinas y femeninas como dos valores contrapuestos y jerarquizados (Barragán et al., 2002; García- Prieto \& Arriazu- Muñoz, 2020). Estas ideas y valores sobre los géneros desencadenan la 
presencia de estereotipos, que explican, en buena parte, por qué un niño tenga menos deseos de jugar con muñecas o a una niña se le dificulte o se niegue a jugar fútbol (Rodríguez, 2020). En síntesis, los estereotipos de género están influenciados por los agentes de socialización (familia, escuela y medios masivos de comunicación), los que promueven un estilo de desarrollo intelectual, afectivo y social en función del sexo, legitimando silenciosamente las diferencias por género. En este sentido, se ha señalado que «en el área de apariencia física, parece mantenerse la típica asociación culturalmente establecida: rosa para chicas y azul para chicos. ...Esta asignación de colores es totalmente arbitraria y promovida culturalmente» (Rodríguez, 2020, p. 69).

La génesis y el origen del género también es un asunto complejo que ha generado múltiples discusiones epistemológicas. De acuerdo con Butler (1998), el género es afectado por la sociedad, pero no puede ser reducido a ninguna esencia biológica, psicológica o cultural. No obstante, dado que es sabido que el factor biológico, psicológico y cultural lo influyen, también se ha planteado que sería un fenómeno biopsicosocial (Mujica, 2021; Scheele \& Barker, 2021). Entre los dos años y medio y tres años, niñas y niños comienzan a diferenciar, separar y catalogar los sexos, fijándose en características externas. Por otro lado, entre los tres y cinco años (la etapa de prescolar), comienzan a creer que el hecho de sentirse niña o niño es algo fijo o estable, imposible de cambiar, básicamente determinado por su sexo (Rodríguez, 2020). Las niñas de acuerdo al género femenino son enseñadas a ser amables y a no responder violentamente, por el contrario, la educación de la masculinidad es ejercer la violencia para resolver conflictos y suprimir sus sentimientos (González, 2005). De este modo, con una fuerte influencia cultural, feminidades y masculinidades tienen diferentes formas ontológicas de ser y parecer (Cobo, 2008). En este sentido, la infancia se representa como una infancia sometida y domesticada (Grabivker, 2019), básicamente a las demandas y construcciones del género. El sistema patriarcal y el adulto-centrismo se establecen como fuerzas relacionales que establecen el dominio social, sobre unos imaginarios en torno a lo que es mayor y menor; bueno y malo; autónomo y dependiente; fuerte y débil (Duarte, 2016).

Esta idea de dominación hacia la infancia y un ausente abordaje del infante como sujeto de derecho (Grabivker, 2019), está asociado a dos principales tensiones. Por un lado, al escepticismo que rodea la posibilidad de que puedan existir infantes con una identidad de género distinta a la que les fue asignada al nacer, es decir, que existan menores de edad que se identifiquen como personas trans (Guerrero \& Muñoz, 2018). Por otro lado, está la transmisión de normas y códigos de género que los adultos transmiten a los menores por medio del juego y, en dicho contexto, los juguetes son especialmente relevantes para la formación de la identidad de género (Álvarez, Carrera-Fernández \& CidFernández, 2017).

Uno de los aspectos más importantes a desarrollar en la infancia es la psicomotricidad, donde, evidentemente, se desarrollan múltiples juegos motores, los cuales pueden estar influenciados, de distinto modo, por una cultura patriarcal y sus estereotipos de género (Bonilla, 2018). Por lo mismo, como bien explicó Cabrera (2020), es posible y necesario repensar el currículum y la didáctica de la educación infantil desde un enfoque feminista que promueva la igualdad de género. En el caso de la psicomotricidad, se ha señalado que es fundamental atender la afectividad de la persona en su etapa infantil, incluyendo sus emociones, sus estados de ánimo o sus actitudes (Aucouturier, 2007). Pues, se debe considerar a la persona de un modo integral o en su totalidad, evitando una mirada fragmentada o reduccionista. Asimismo, una educación psicomotriz ha de considerar el factor cultural (Pastor-Pradillo, 2002), ya que existen múltiples elementos simbólicos de la sociedad que participan de aquel proceso pedagógico. De lo contrario, si solamente se atienden factores psicológicos y conductuales, se asumiría una racionalidad educativa técnica, conductista y positivista (Díaz, 2005; Gimeno-Sacristán, 2002). Racionalidad que, por lo demás, todavía está muy presente en el sistema educativo chileno (Matus, 2016; Moreno \& Gamboa, 2014; Moreno, Gamboa \& Poblete, 2014; Mujica \& Inostroza, 2020; Mujica \& Toro, 2019; Oliva \& Gascón, 2016).

Con base en lo anteriormente expuesto, en el presente ensayo se tiene por objetivo analizar desde una perspectiva crítica y de género cuatro documentos ministeriales chilenos sobre psicomotricidad y corporalidad en edad infantil. En concreto, los siguientes documentos: a) orientaciones teóricas y técnicas para el manejo de sala de psicomotricidad (Araya, 2017); b) experiencias de aprendizaje sobre corporalidad y movimiento. Libro 1 (MINEDUC, 2011a); c) experiencias de aprendizaje sobre corporalidad y movimiento. Libro 2 (MINEDUC, 2011b); y d) bases curriculares de Educación Parvularia (MINEDUC, 2018). Sobre el último documento, de las bases curriculares, se analizó el núcleo corporalidad y movimiento. 


\section{Psicomotricidad y neutralidad en torno al género}

Los documentos analizados en torno a la psicomotricidad y la corporalidad en edad infantil reflejan, en parte, una orientación hacia una teoría pedagógica posmoderna (Mujica, 2020a; Sicilia, 2012), trascendiendo, por ejemplo, la concepción ontológica dualista platónico-cartesiana que fue hegemónica en la época moderna. La misma lógica que objetivó o cosificó la corporalidad, y se desentendió de los aspectos subjetivos del ser humano en torno a su formación humana integral (física, motriz, emocional, social, psicológica, intelectual, ética). Aquel enfoque más integral se niega a fragmentar radicalmente al ser humano $y$, específicamente, en la edad infantil, como se puede apreciar en la siguiente cita:

Si se entiende la escuela desde una amplia perspectiva en su labor formativa, podemos afirmar que su misión en la etapa inicial y primaria, no consiste sólo en transmitir conocimientos, aplicar y enseñar técnicas para el aprendizaje de contenidos, sino, ante todo, ser un espacio para la formación de personas. Empero, la escuela suele desestimar al cuerpo en la formación integral del ser humano, la dicotomía mente/cuerpo, intelectual/ práctico, o peor aún, principal/secundario, marca el desinterés por incluir al cuerpo en esta tarea. Lo anterior es posible debido a la segmentación de saberes: por un lado, se encuentran las asignaturas que se ocupan del cuerpo y, por el otro, aquellas que se ocupan de la mente. Esta contradicción cuerpo/mente ha permitido la configuración de un imaginario escolar donde prevalece la superioridad de lo mental sobre lo corporal (MINEDUC, 2017, p. 14).

Se ha mencionado que estos documentos ministeriales reivindicaban parcialmente la teoría pedagógica posmoderna, puesto que se reconoció que carece de una mirada socio-crítica del fenómeno educativo. En este sentido, como bien explica Torres (2002), tendría implícito un fuerte acento de la neutralidad positivista, conductista y cientificista heredado de la filosofía de la modernidad (Matus, 2016). O, con base en Giroux (1990), una tendencia pedagógica reproductiva. Es decir, una tendencia a desnaturalizar o invisibilizar el sentido político, filosófico y cultural de la educación (Freire, 2011, 2012; Gimeno-Sacristán, 2002; Hirsh \& Rio, 2015; Kirk, 2010; Lawson, 2008, 2018, 2019; Moreno, Gamboa y Poblete, 2014; Mujica, 2020b). De hecho, aquello sería coherente con la hegemonía del enfoque positivista que todavía existe en el currículum chileno, basado en una pedagogía por objetivos que fragmenta excesivamente el aprendizaje, en su supuesta neutralidad ideológica, en la estandarización educativa y en su obsesión por la eficiencia (Moreno \& Gamboa, 2014; Moreno, Rivera \&Trigueros, 2014; Mujica, 2020c; Mujica \&Toro, 2019; Oliva \& Gascón, 2016).

En esta epistemología pedagógica, relativamente moderna, presente en los documentos ministeriales analizados, se aborda la psicomotricidad y la corporalidad desde una mirada técnica que desglosa de diferentes formas el factor motriz y expresivo del ser humano. Pero, como suele hacerse en los modelos curriculares influenciados por la mirada tecnológica (Monzón, 2010), lo hacen sin hacer mención a buena parte de los conflictos socioculturales, filosóficos y políticos que influyen en el tema. En este sentido, no se aprecian elementos significativos de la teoría feminista que pretenda avanzar hacia una igualdad de género real y situada en función de las dificultades de cada contexto (Jiménez, 2011; Martínez, 2016; Martínez \& Ramírez, 2017). En relación a esta invisibilización de los conflictos que existen en torno a la cultura patriarcal, es que cobra mayor sentido un reciente análisis sobre las políticas educativas chilenas de género, el cual concluyó lo siguiente:

Con respecto a la perspectiva chilena, se concluye que el MINEDUC en Chile no cuenta con una normativa específica para tratar los temas de género en cada uno de los niveles educativos, aunque sí hacen recomendaciones relacionadas a los planes de convivencia, los cuales contemplan orientaciones generales para tratar la temática género, dejando autonomía a los centros. De esta forma, se constata que el país sudamericano se encuentra en vías de institucionalizar la perspectiva de género (...) Dado que en Chile ha aumentado la conciencia sobre las políticas educativas de género, es necesario que no sean entendidas desde una mirada reduccionista, tanto en la teoría como en la práctica; es decir, es muy importante que se amplíe la conciencia sobre el fenómeno aludido, sobre todo, para que incluya una mirada integral a las políticas educativas en dicha temática, reconociendo aspectos estructurales, curriculares y socioculturales. De este modo, se promoverían políticas educativas que reconozcan la complejidad del asunto del género y las profundas raíces que sostienen las desigualdades en la materia (Concha y Mujica, 2021, pp. 12-13).

La invisibilización de los conflictos que existen en torno a la cultura patriarcal, también está presente en la omisión en torno a los estereotipos de género que se reflejan en la actividad lúdica de la niñez (Puerta \& 
González, 2015; Rodríguez, 2020; García- Prieto \& Arriazu- Muñoz, 2020). Siendo el juego un proceso psicológico que contribuye en la construcción de la identidad de género y la incorporación de roles, valores, actitudes y comportamientos que perpetúan los estereotipos establecidos (García- Prieto y Arriazu- Muñoz, 2020). Por ejemplo, algunos estudios que se han propuesto observar y analizar el juego socio dramático infantil (Puerta \& González, 2015; García- Prieto \& Arriazu- Muñoz, 2020), concluyen que las niñas y los niños desarrollan juegos muy distintos, orientándose las niñas a representar roles de hijas mayores, mamás, bebés y princesas bellas, con el imperativo de realizar tareas domésticas y de cuidado a bebés. Por el contrario, los niños suelen preferir ser superhéroes, monstruos y animales basándose en la fuerza física, la violencia y los poderes. De este modo, se manifiesta una socialización diferencial en torno al género, la cual también tendría un fuerte impacto en la elección de juguetes en función de los estereotipos hegemónicos de masculinidad y feminidad (Álvarez et al., 2017). Por ello, en el contexto analizado, pareciera ser que en la educación motriz infantil se continúan normalizando y perpetuando dichas construcciones sociales sexistas de dominación, profundamente arraigadas en el acervo cultural.

Por otro lado, en los diferentes documentos ministeriales analizados se puede apreciar una tendencia a visibilizar el género femenino, que ha sido uno de los más perjudicados por la cultura patriarcal (Bourdieu, 2000; Carrillo, 2017; Curieses, 2017), haciendo alusión constantemente a niños y niñas, entendiendo que se refiere a un alumnado infantil. Esto se puede apreciar en la siguiente cita:

Las experiencias de movimiento y de conocimiento de la corporalidad que progresivamente adquieran los niños y las niñas a través de sus exploraciones o en diferentes juegos les facilitarán un proceso gradual de organización psicomotriz para funcionar de manera más autónoma, desarrollando una identidad más fortalecida y armónica. El niño y la niña aprenden con y desde su cuerpo, esta condición determinará «el cómo» van a integrar nuevos conocimientos sobre múltiples aspectos del mundo que los rodea y sobre sí mismo. Todos los niños/as utilizan su motricidad no sólo para moverse, para desplazarse, para tomar los objetos o para expresar sus emociones sino, fundamentalmente, para ser, para aprender a aprender y para organizar su pensamiento (MINEDUC, 2011b, p. 5).

Aquella tendencia de visibilizar el género femenino es, sin duda, una buena acción para avanzar en la equi- dad de género, pero, por ningún motivo, es un acto muy significativo para una mirada sociocrítica que trascienda la lógica neutral del positivismo. En otras palabras, se reconoce como un acto muy superficial para un problema que es muy complejo y tiene variadas capas de profundidad que pueden ser sometidas a un análisis crítico. Por ejemplo, para avanzar hacia una política educativa que comprenda la complejidad del fenómeno feminista y de género, se ha de considerar, por lo menos, los siguientes elementos en una programación pedagógica de psicomotricidad y formación corporal: a) el alumnado puede no identificarse con un género binario, de modo que no sería representado con niño o niña; b) estudiantes transexuales que podrían ser víctimas de discriminación por parte del entorno; c) estereotipos de género asociados a la vestimenta que puede ser utilizada para actividades motrices; d) juegos simbólicos que pueden ser discriminadores hacia las diferentes manifestaciones de género; e) materiales psicomotrices estereotipados por la perspectiva cultural patriarcal; f) emociones de los estudiantes posiblemente afectadas culturalmente por la perspectiva patriarcal, y que se manifiestan en la expresión motriz en el aula; y g) los niveles de habilidad motriz podrían estar influidos por la cultura patriarcal que favorece ciertas actividades motrices en niños y otras en niñas, aspectos que fueron mencionados en un anterior apartado.

Además de lo mencionado, cabe considerar que, en Chile, muchas actividades psicomotrices se desarrollan en instituciones que integran la educación infantil con otras edades más avanzadas, como la edad adolescente. $\mathrm{Al}$ respecto, un reciente estudio reconoció que existen reglamentos internos de centros educativos chilenos que estereotipan la corporalidad (Mujica, 2019), de modo que el alumnado infantil podría tener la influencia de una cultura escolar sexista.

\section{Psicomotricidad, corporalidad e influencia positivista}

La formación pedagógica de psicomotricidad y corporalidad que se presenta en los documentos ministeriales analizados, como bien se explica en el apartado anterior, no es totalmente positivista, ya que sí asume una postura filosófica frente a conceptos que fueron por mucho tiempo hegemónicos. Sin embargo, no se aprecia una discusión crítica de múltiples aspectos culturales que son determinantes, contingentes y polémicos desde la perspectiva crítica. A su vez, el dualismo cuerpomente contra el que se enfrenta es bastante popular o 
común entre los especialistas de la motricidad humana. En este sentido, no es un aspecto muy controversial y original, aunque sí muy influyente en el sistema educativo chileno y mundial.

La influencia positivista en los documentos analizados también se acusa por las fuentes bibliográficas que han servido de referente, sobre todo, en la mayor presencia de fuentes técnicas en el documento de Araya (2017) y MINEDUC (2018), como en la ausencia de fuentes bibliográficas en los otros dos documentos. Por mayor presencia de fuentes técnicas, se hace referencia a autorías que no se enmarcan en una línea de pedagogía o filosofía crítica. Es decir, al igual que el estilo de aquellos documentos en torno a la psicomotricidad y la corporalidad, optan por una línea discursiva que no problematiza las influencias políticas y culturales, así como las lógicas de poder que existen en torno a la educación (Apple, 2015). Esto mismo se refleja en algunos programas de psicomotricidad, los cuales parecen entrenamientos motrices, ajenos a un proceso de formación pedagógica. Es decir, se aprecia una lógica conductista donde hay un aprendizaje pasivo por parte del alumnado. Por otro lado, cabe destacar el rango etario de la etapa infantil, pudiendo ser propicia para una educación psicomotriz adoctrinadora, donde no se estimula la participación activa y autónoma del alumnado, bajo la consigna de que no son muy capaces de asumir procesos autónomos. En relación a una enseñanza conductista en edad infantil, estaría basada, en buena parte, en «la repetición constante de instrucciones, la asignación de tareas a desarrollar, la imitación de conductas, el control y monitoreo del trabajo en clase, el ejercicio y práctica de habilidades, la corrección y la sanción, y los acuerdos de clase establecidos por el docente» (Acosta, Castaño \& Vega, 2015, p. 118).

Cuando existe un enfoque tecnicista en torno a una temática pedagógica, los autores referenciados suelen ser presentados, en términos metafóricos, como los dueños de la verdad sobre lo que se aborda. Asimismo, las personas que son autoras de un documento con respaldo ministerial también suelen asumir dicha postura. En el caso de la psicomotricidad y la corporalidad, los documentos incluyen perspectivas teóricas que, al igual como sucedió con el dualismo platónico-cartesiano en el pasado, son expuestas como un modelo a reproducir sin mayor cuestionamiento de sus premisas. Por ejemplo, en el documento de Araya (2017) en torno a los principios temporales de la psicomotricidad, la autora señaló que «la sesión se organiza en fases diferenciadas que se ejecutan siempre en el mismo orden y con la misma duración, ya que constituyen el elemento estructurante y estructurador de la sesión de juego» (p. 38). Entonces, cabe preguntarse, ¿es posible realizar otra organización? ¿por qué tener que fragmentar siempre con el mismo orden? ¿qué lógica es la que predomina en esa instrucción? Evidentemente es una lógica conductista, pero esta vez no aplicada exclusivamente al alumnado, sino que al profesorado encargado de aplicar y reproducir dichas indicaciones. Posteriormente, la misma autora expone diferentes fases en torno a la temporalidad, dando indicaciones de lo que sería correcto e incluyendo al final de la instrucción una cita textual de un autor que utiliza frecuentemente sobre la temática. Lo interesante es que no se realizan apreciaciones o discusiones sobre la cita, sino que se deja a modo de respaldo de lo señalado. Esto podría querer transmitir, muy probablemente, que dado que existe una fuente bibliográfica no puede ser puesto en duda. Por ello, nos encontramos frente a una lógica academicista que no invita al profesorado a reflexionar sobre la temática y generar sus propias conclusiones sobre la mejor forma de educar la psicomotricidad y la corporalidad. En buena parte, lo anterior representaría una lógica bancaria de la educación (Freire, 2011), donde el profesorado cumpliría un rol pasivo frente a la información ministerial. Es decir, una lógica acrítica y anti emancipadora, que no promueve una educación de buena calidad. Frente a esta realidad, se considera que una educación del profesorado emancipadora debería reconocer, en parte, lo siguiente:

La emancipación es un tipo de pensamiento reflexivo, profundo y auto cuestionador que implica un esfuerzo sostenido y una fuerte revisión del propio pensamiento y sus supuestos epistemológicos e históricos, yendo más allá de la aceptación de un conjunto de saberes y valoraciones universales e incuestionadas provenientes de la teoría previa o de la cultura dominante (Bazán, 2019, p. 18).

Considerando a la emoción como un componente importante de la educación psicomotriz y corporal, es preciso señalar que un estudio de las nuevas bases curriculares de educación parvularia en Chile concluyó que existe un abordaje crítico y otro acrítico de la afectividad (Mujica y Toro, 2019). En el caso del núcleo Corporalidad y Movimiento de las bases curriculares, se reconocen objetivos de aprendizaje afectivo acríticos, positivistas y psicologistas. Por ejemplo, en el primer nivel (sala cuna) se declaran los siguientes dos objetivos de aprendizajes transversales que tendrían dichas características: «1. Manifestar su agrado, al sentirse cómodo, 
seguro y contenido corporalmente. (...) 3. Manifestar interés y satisfacción al moverse libremente en situaciones cotidianas y lúdicas» (MINEDUC, 2018, p. 62). A simple vista aquellos objetivos podrían parecer adecuados y deseables, pero un análisis profundo de ellos reflejaría que existen algunos supuestos epistemológicos dominantes de una cultura psicologista y positivista (Marina, 2005, 2009; Mujica \& Orellana, 2021; Prieto, 2018), lo cual, por supuesto, es un análisis que ha de ser discutido.

En primer lugar, cabe preguntarse, ¿puede ser un estado sentimental o una emoción un objetivo aprendizaje que dependa principalmente de la voluntad de la persona? En función de que la emoción es bastante influenciada por las experiencias de vida, las oportunidades, el entorno socioeconómico, el entorno político, el entorno moral, el entorno filosófico, la cultura en general, la biología, la psicología, el tipo de convivencia, el estilo de comunicación, etc., podemos concluir que la emoción no es algo que dependa en todos los casos y principalmente de la propia persona. ¿Qué sucedería si hay un alumno que tiene una discrepancia constante con el trato del profesorado en las actividades motrices y corporales? Frente a esa situación, ¿estaría mal que el alumnado sienta desagrado e insatisfacción? Dicho de otro modo, ¿no se habría logrado el objetivo de aprendizaje transversal? Podrían mencionarse múltiples situaciones que no susciten agrado en el alumnado y que nos lleven a los mismos cuestionamientos. En los objetivos de aprendizajes transversales mencionados anteriormente, del primer nivel en el núcleo Corporalidad y Movimiento (MINEDUC, 2018), no hay ningún objetivo de aprendizaje que incluya una emoción negativa para el bienestar subjetivo. Aquello refleja la aplicación acrítica de la teoría del bienestar subjetivo a la educación, lo que en muchos trabajos académicos se ha cuestionado por su interpretación individualista, cientificista, utilitaria y neoliberal de los afectos (Illouz, 2007; Manrique, 2015; Mujica, 2020b).

\section{Conclusiones y aplicaciones prácticas}

En función del objetivo planteado en el presente ensayo, analizar desde una perspectiva crítica y de género cuatro documentos ministeriales chilenos sobre psicomotricidad y corporalidad en edad infantil, se concluye que existen algunas perspectivas epistemológicas tradicionales predominantes e implícitas en los textos analizados. En primer lugar, se aprecia la ausencia de un abordaje crítico del género en la formación psicomotriz y corporal propuesta, lo que genera una educación reproductiva y técnica en dicha materia. Se aprecia, en buena parte, una mirada neutral frente a la influencia de la cultura patriarcal en la educación infantil. Por ello se aprecia que todavía no existe una influencia significativa de los estudios sobre las mujeres, de género y/o feminismo académico que se han desarrollado en los últimos años, los cuales han nacido bajo la idea de promoción de nuevos saberes alejados del orden androcéntrico (García, 2017). Representando una oportunidad para entender los procesos desde otro prisma que deconstruye dicho orden cultural. No obstante, se reconoció la intencionalidad de visibilizar el género femenino en el lenguaje verbal de los documentos ministeriales, lo cual podría ser el mayor acto de promover la igualdad de género en torno a la psicomotricidad que estaría presente en dichos documentos.

La predominancia de una racionalidad curricular acrítica o técnica en torno a la psicomotricidad, la corporalidad y el género, se asociaría a la influencia de la filosofía positivista en la cultura pedagógica chilena, la cual, en general, no promueve los pensamientos, las interpretaciones, las actitudes y las acciones críticas. En otras palabras, no promueve una educación infantil emancipadora. De este modo, se estimula que el profesorado de educación infantil asuma una actuación pasiva frente a las concepciones teóricas que son hegemónicas o dominantes en la cultura. Asimismo, se modela una postura ética indiferente a las injusticias sociales y a las posibilidades de mejorar la calidad de la cultura desde la educación formal. En el caso del género, se mostraría una notable indiferencia ante los aportes de la teoría feminista en los procesos pedagógicos, sobre todo, frente a los estereotipos de género androcéntricos.

A modo de aplicaciones prácticas, se considera fundamental que este análisis crítico pueda ser debatido entre docentes de educación infantil y universitarios asociados a la formación pedagógica en edad infantil. Asimismo, se hace necesario trabajar en la elaboración y difusión de estrategias de aprendizaje basadas en el modelo coeducativo y sustentadas en la teoría feminista, pues un modelo de educación infantil coeducativo permite educar en el respeto a la diversidad, apoyándose en los principios de equidad y cuidado (Mañas-Viejo, 2018). Además, sería relevante que el ministerio de educación en Chile pueda ser interpelado por el fuerte acento técnico y positivista en la racionalidad curricular de sus textos. 


\section{Futuras líneas de investigación}

En este apartado se expondrán dos líneas de investigación. La primera sería con el profesorado de educación infantil, referido a sus teorías implícitas sobre el género, la psicomotricidad y la corporalidad. Esta línea de estudio sería de corte empírico y de corte cualitativo, con la finalidad de profundizar en contextos situados sobre las interpretaciones y los significados del fenómeno pedagógico aludido.

La segunda línea de investigación, también sería de corte empírico, pero en torno a intervenciones curriculares y didácticas en educación infantil que aborden la educación infantil psicomotriz y corporal desde una perspectiva emancipadora en torno al género.

\section{Referencias}

Acosta, P., Castaño, P., \& Vega,A. (2015). Los modelos de enseñanza y su aporte a la formación en valores en el nivel preescolar. En L. Castillo (Ed.), Transformar la docencia. Investigaciones sobre formación en valores, resolución de problemas y lectura crítica (pp. 111-122). Bogotá: Universidad de La Salle.

Álvarez, N., Carrera-Fernández, M.V., \& Cid-Fernández, X. M. (2017). juegos de niñas y juegos de niños? la influencia de los estereotipos de género en la elección de juguetes. Revista de estudios de investigación en psicología y educación, Extr.(05), 1-4. doi: 10.17979/reipe.2017.0.05.2830

Apple, M. (2015). Conocimiento, poder y educación: sobre un saber académico/activista. Entramados: Educación y Sociedad, 2, 29-39.

Araya, E. (2017). Orientaciones teóricas y técnicas para el manejo de sala de psicomotricidad. Santiago de Chile: MINEDUC.

Aucouturier, B. (2007). Los fantasmas de acción y la práctica psicomotriz ( $3^{\mathrm{a}} \mathrm{ed}$.). Barcelona: GRAÓ.

Barragán, F., Sanz, J., Doblas, J. J., Hernández, B., Miguel, L., \& Guerra, M.I. (2002). Educación en valores y género. Sevilla: Diada editora.

Bonilla, N. (2018). Estereotipos de género en los juegos tradicionales infantiles (Tesis de pregrado no publicada). UniversidadTécnica de Ambato, Ecuador.

Bazán, D. (2019). Calidad de la educación y emancipación. Opiniones de un pedagogo. Santiago de Chile: Mutante editores.

Bourdieu, P. (2000). La dominación masculina. Barcelona: Anagrama.

Butler, J. (1998). Actos performativos y constitución del género: un ensayo sobre fenomenología y teoría feminista. Debate Feminista, 18, 296-314.

Cabrera, A. (2020). Estereotipos de género en educación infantil: diseño de una propuesta formativa como caso práctico (Tesis de pregrado no publicada). Universidad de Sevilla, España.

Carrillo, I. (2017). Los nudos del género. Apuntes para la formación ética de educadoras y educadores. Revista Internacional de
Educación para la Justicia Social, 6(2), 29-48.

Cobo, R. (2008). Repensando la democracia: Mujeres y Ciudadanía. En R. Cobo, Educar en la ciudadanía. Perspectivas Feministas (pp. 19-52). Madrid: Catarata.

Concha, R., \& Mujica, F. (2021). Sobre las políticas educativas de género: perspectiva internacional y chilena. Revista Dilemas Contemporáneos: Educación, Política y Valores, 8(Ed. Especial), 118. doi: $10.46377 /$ dilemas.v8i.2760

Curieses, P. (2017). Por una escuela inclusiva. Las fronteras del género. Revista Internacional de Educación para la Justicia Social, 6(2), 63-79.

Díaz, J. (2005). La evaluación formativa como instrumento de aprendizaje en Educación Física. Barcelona: Inde.

Duarte, K. (2016). Genealogía del adultocentrismo. La construcción de un patriarcado adultocéntrico. En K. Duarte, \& C. Álvarez, Juventudes en Chile. Miradas de jóvenes que investigan (pp. 17-47). Santiago de Chile: Edición de la Facultad de Ciencias Sociales Universidad de Chile.

Freire, P. (2011). Pedagogía del oprimido ( $2^{\mathrm{a}}$ ed. $5^{\mathrm{a}}$ reimpresión). México, D.F: Siglo xxi

Freire, P. (2012). Pedagogía de la Indignación. México, D.F: Siglo xxi.

García-Prieto, I., \& Arriazu- Muñoz, R. (2020). Estereotipos de género en educación infantil: Un estudio de caso desde la perspectiva sociocultural. Zero a Seis, 22(41), 4-30. doi: $10.5007 / 1980-4512.2020 \mathrm{v} 22 \mathrm{n} 41 \mathrm{p} 4$

García, M. (2017). La perspectiva de género en la formación incial del profesorado de educación infantil. Notas sobre una investigación realizada entre el alumnado del grado de magisterio de educación infantil de la Universidad de Cantabria. exaequo, 4357. doi: https://doi.org/10.22355/exaequo.2017.36.03

Gimeno-Sacristán, J. (2002). La pedagogía por objetivos: obsesión por la eficiencia (11 $\mathrm{a}$ ed.). Madrid: Morata.

Giroux, H. (1990). Losprofesores como intelectuales. Barcelona: Piados.

González, M. (2005). Del sexismo a la igualdad de oportunidades en la educación. En L. Fernández, Género, valores y sociedad, una propuesta desde Iberoamérica (pp. 73-94). Madrid: Octaendro OEI.

Grabivker, M. (2019). ¿A qué nos referimos cuando hablamos y escribimos sobre infancia en la actualidad? En R. Gamboa, G. Jiménez, \& C. Cacciuttolo (Eds.), Motricidad Infantil. Bases y lineamientos para re-crear con los niños y niñas trayectorias de placer y realización vital (pp. 17-25).Valparaíso: Ediciones Universitarias deValparaíso-Pontificia Universidad Católica deValparaíso.

Guerrero, S., \& Muñoz, L. (2018). Epistemologías transfeministas e identidad de género en la infancia: del esencialismo al sujeto del saber. Revista Interdisciplinaria de estudios de género del Colegio de México, 4, 2-31. doi: 10.24201/eg.v4i0.168

Hirsch, D. \& \& Rio,V. (2015).Teorías de la reproducción y teorías de la resistencia: una revisión del debate pedagógico desde la perspectiva materialista. Foro de Educación, 13(18), 69-91. doi: 10.14516/fde.2015.013.018.004

Illouz, E. (2007). Intimidades congeladas. Las emociones en el capitalismo. Buenos Aires: Katz. 
Jiménez, C. (2011). Educación, Género e Igualdad de Oportunidades. Tendencias Pedagógicas, 18, 55-83.

Kirk, D. (2010). Physical Education Futures. NewYork: Routledge.

Lawson, H. (2009). Paradigms, exemplars and social change. Sport, Education and Society, 14(1), 97-119. doi: 10.1080/ 13573320802615247

Lawson, H. (2018). Physical Education in the industrial age school. En H. Lawson (Ed.), Redesigning Physical Education: An equity agenda in which every child matters (pp. 23-40). New York: Routledge.

Lawson, H. (2019). The Physical Education System as a Consequential Social Determinant. Quest, 72(1), 72 84. doi:10.1080/00336297.2019.1627224

Lévi-Strauss, C. (1995). Antropología estructural. Paidós.

Lévi-Strauss, C. (2019). Raza y cultura (9a ed.). Cátedra.

Manrique, R. (2015). La cuestión de la inteligencia emocional. Revista de la Asociación Española de Neuropsiquiatría, 35(128), 801-814. doi: 10.4321/S0211-57352015000400008

Mañas-Viejo, C. (2018). Coeducación: imprescindible en la formación de la Educación Infantil. El caso de la Psicología Evolutiva. Entorno(66), 113-123. doi:http://dx.doi.org/ 10.5377/entorno.v0i66.6731

Marina, J. A. (2005). Precisiones sobre la educación emocional. Revista Interuniversitaria del Profesorado, 54, 27-44.

Marina, J. A. (2009). El laberinto sentimental ( $9^{\mathrm{a}} \mathrm{ed}$.). Barcelona: Anagrama.

Martínez, I. (2016). Construcción de una pedagogía feminista para una ciudadanía transformadora y contra-hegemónica. Foro de educación, 14(20). 129-151. doi: 10.14516/ fde.2016.014.020.008

Martínez, I. y Ramírez, G. (2017). Des-patriarcalizar y Des-colonizar la Educación. Experiencias para una Formación Feminista del Profesorado. Revista Internacional de Educación para la Justicia Social (RIEJS), 6(2), 81-95. doi: 10.15366/ riejs2017.6.2.005

Matus, C. (2016). Los usos del afecto en el currículo escolar. Educação em Revista, 32(2), 111-130. doi: 10.1590/01024698153449

MINEDUC. (2011a). Experiencias de aprendizaje sobre corporalidad y movimiento.Libro 1. Santiago: República de Chile.

MINEDUC. (2011b). Experiencias de aprendizaje sobre corporalidad y movimiento. Libro 2. Santiago: República de Chile.

MINEDUC. (2018). Bases curriculares educación parvularia. Santiago: República de Chile.

Monzón, L.A. (2010). El currículo como mediación cultural: una perspectiva hermenéutico-analógica. Revista Latinoamericana de Estudios Educativos, 40(2), 37-58.

Moreno, A., Gamboa, R., \& Poblete, C. (2014). La educación física en chile: análisis crítico de la documentación ministerial. Revista Brasileira de Ciências do Esporte, 36(2), 411 427.

Moreno, A., \& Gamboa, R. (2014). Dictadura chilena y sistema escolar: «a otros dieron de verdad esa cosa llamada educación». Educar em Revista, 51, 51-66. doi: 10.1590/S0104-
40602014000100005.

Moreno,A., Rivera, E., \& Trigueros, C. (2014). Sistema de medición de la calidad de la educación física chilena: Un análisis crítico. Movimento, 20(1), 145-167. doi: 10.22456/19828918.41485

Mujica, F. (2019). Reglamento sexista en los centros de educación escolar en Chile. CPU-e. Revista de Investigación Educativa, 29, 87-107. doi: 10.25009/cpue.v0i29.2634

Mujica, F. (2020a). El término Educación Física en la postmodernidad: contribución de algunas perspectivas fenomenológicas. Retos. Nuevas Tendencias en Educación Física, Deportes y Recreación, 38, 795-801. doi: 10.47197/ retos.v38i38.73011

Mujica, F. (2020b). Emociones morales en educación: análisis del enfado en el contexto neoliberal. Revista Educación, Política $Y$ Sociedad, 5(2), 33-49. doi: 10.15366/reps2020.5.2.002

Mujica, F. (2020c). Análisis crítico del currículo escolar en Chile en función de la justicia social. Revista Electrónica Educare, 24(1), 472-485. doi: 10.15359/ree.24-1.25

Mujica, F. (2021). Filosofía y Ser Humano. Reflexiones para la Ciudadanía. Santiago de Chile:Trayecto.

Mujica, F., \& Inostroza, C. (2020). Políticas educativas, evaluaciones estandarizadas y formación escolar integral en Chile: análisis de la desvalorización a diferentes materias del currículo. Dilemas Contemporáneos: Educación, Política y Valores, 7(2), 1-20.

Mujica, F, \& Orellana, N. (2021). El giro emocional de la educación. Santiago de Chile: Forja.

Mujica, F., \& Toro, M.A. (2019). Formación afectiva en la educación parvularia de Chile. Cuadernos de Investigación Educativa, 10(2), 57-71. doi: 10.18861/cied.2019.10.2.2907

Oliva, M.A., \& Gascón, F. (2016). Estandarización y racionalidad política neoliberal: Bases curriculares de Chile. Cadernos $\mathrm{Ce}$ des, 36(100), 301-318. doi: 10.1590/cc010132622016171349

Pastor-Pradillo, J. L. (2002). Fundamentación conceptual para una intervención psicomotriz en Educación Física. Barcelona: Inde.

Prieto, M. (2018). La psicologización de la educación: implicaciones pedagógicas de la inteligencia emocional y la psicología positiva. Educación XX1, 21(1), 303-320. doi: 10.5944/ educXX1.16058

Puerta, S., \& González, E. M. (2015). Reproducción de los estereotipos de género en Educación Infantil a través los juegos y juguetes. Investigación en la Escuela, 85, 63-74. doi: 10.12795/ IE.2015.i85.05

Rodríguez, B. (2020). Evaluación de los estereotipos de género en educación infantil. Know and Share Psychology, 1(3), 63-70. doi: 10.25115/kasp.v1i3.3765

Scheele, J., \& Barker, M. (2021). Género. Una guía gráfica. Melusina. Sicilia, Á. (2012). Educación Física y transformación social: implicaciones desde una epistemología posmoderna. Estudios pedagógicos (Valdivia), 38(especial), 47-65. doi: 10.4067/ S0718-07052012000400004

Torres, J.A. (2002). ¿Existe un currículum postmoderno? De las teorías curriculares de la modernidad al currículum postmoderno. Revista Innovación Educativa, 12, 197-208. 